\title{
Flow polytopes of partitions
}

\author{
Karola Mészáros* Connor Simpson Zoe Wellner \\ Department of Mathematics \\ Cornell University \\ Ithaca, NY, USA \\ karola@math.cornell.edu cgs93@cornell.edu zaw5@cornell.edu \\ Submitted: Aug 22, 2018; Accepted: Mar 1, 2019; Published: Mar 22, 2019 \\ (C) The authors. Released under the CC BY-ND license (International 4.0).
}

\begin{abstract}
Recent progress on flow polytopes indicates many interesting families with product formulas for their volume. These product formulas are all proved using analytic techniques. Our work breaks from this pattern. We define a family of closely related flow polytopes $\mathcal{F}_{(\lambda, \mathbf{a})}$ for each partition shape $\lambda$ and netflow vector $\mathbf{a} \in \mathbb{Z}_{>0}^{n}$. In each such family, we prove that there is a polytope (the limiting one in a sense) which is a product of scaled simplices, explaining their product volumes. We also show that the combinatorial type of all polytopes in a fixed family $\mathcal{F}_{(\lambda, \mathbf{a})}$ is the same. When $\lambda$ is a staircase shape and $\mathbf{a}$ is the all ones vector the latter results specializes to a theorem of the first author with Morales and Rhoades, which shows that the combinatorial type of the Tesler polytope is a product of simplices.
\end{abstract}

Mathematics Subject Classifications: 52B20, 05C21

\section{Introduction}

The Catalan numbers, $C_{n}=\frac{1}{n+1}\left(\begin{array}{l}n \\ 2\end{array}\right), n \in \mathbb{Z}_{\geqslant 0}$, are well known for counting a plethora of combinatorial objects; see [6, Ex. 6.19] for hundreds of interpretations. Naturally then, if an integer polytope has volume divisible by a product of consecutive Catalan numbers, one would hope for a combinatorial explanation of such a phenomenon. The latter sentiment ran into obstacles with several flow polytopes, namely the (type A) Chan-Robbins-Yuen polytope [2], its type $\mathrm{C}$ and $\mathrm{D}$ generalizations [4], as well as the Tesler polytope [5]. Our work is inspired by the Tesler polytope (which is the flow polytope of the complete graph with netflow vector all ones) $\mathcal{F}_{K_{n+1}}(\mathbf{1})$, which we explain below can be associated with a staircase partition. Two of the main results known about $\mathcal{F}_{K_{n+1}}(\mathbf{1})$ are as follows. We define $\mathcal{F}_{K_{n+1}}(\mathbf{1})$, and flow polytopes in general, in Section 2.

\footnotetext{
*Mészáros is partially supported by a National Science Foundation Grant (DMS 1501059).
} 
Theorem 1. [5, Theorem 1.9] The normalized volume of the Tesler polytope $\mathcal{F}_{K_{n+1}}(\mathbf{1})$ equals

$$
\begin{aligned}
\operatorname{vol} \mathcal{F}_{K_{n+1}}(\mathbf{1}) & =\frac{\left(\begin{array}{c}
n \\
2
\end{array}\right) ! \cdot 2^{\left(\begin{array}{c}
n \\
2
\end{array}\right)}}{\prod_{i=1}^{n} i !} \\
& =\left|S Y T_{(n-1, n-2, \ldots, 1)}\right| \cdot \prod_{i=0}^{n-1} C_{i},
\end{aligned}
$$

where $C_{i}$ is the $i^{\text {th }}$ Catalan number and $\left|S Y T_{(n-1, n-2, \ldots, 1)}\right|$ is the number of standard Young tableaux of staircase shape $(n-1, n-2, \ldots, 1)$.

Theorem 2. [5, Corollaries 2.8 \& 2.9] The face poset of the Tesler polytope $\mathcal{F}_{K_{n+1}}(\mathbf{1})$ is isomorphic to the face poset of the Cartesian product of simplices $\Delta_{1} \times \Delta_{2} \times \cdots \times \Delta_{n-1}$. In particular, the h-polynomial of the Tesler polytope $\mathcal{F}_{K_{n+1}}(\mathbf{1})$ is the Mahonian distribution

$$
\sum_{i=0}^{\left(\begin{array}{c}
n \\
2
\end{array}\right)} h_{i} x^{i}=[n] !_{x}=(1+x)\left(1+x+x^{2}\right) \cdots\left(1+x+x^{2}+\cdots+x^{n-1}\right) .
$$

For each partition $\lambda$ and vector a we construct a family of flow polytopes $\mathcal{F}_{(\lambda, \mathbf{a})}$, which we define in Section 2.2. The Tesler polytope $\mathcal{F}_{K_{n+1}}(\mathbf{1})$ belongs to $\mathcal{F}_{((n-1, n-2, \ldots, 1), \mathbf{1})}$.

If $\lambda=\left(\lambda_{1}, \ldots, \lambda_{k}\right)$ is a partiton, let $\ell(\lambda):=k$ and let $|\lambda|:=\sum_{i=1}^{k} \lambda_{k}$. For a natural number $n$, let $[n]=\{1,2, \ldots, n\}$. We prove the following general theorems about the families $\mathcal{F}_{(\lambda, \mathbf{a})}$. The limiting polytope $\mathcal{F}_{(\lambda, \mathbf{a})}^{\lim }$ is defined in Section 3.

Theorem 8. Let $\lambda$ be a partition, $n \geqslant \lambda_{1}+\ell(\lambda)$, and $\mathbf{a} \in\left(\mathbb{Z}_{>0}\right)^{n}$ a vector of positive integers. The limiting polytope of $\mathcal{F}_{(\lambda, \mathbf{a})}$ is integrally equivalent to a product of scaled simplices $a_{1} \Delta_{\lambda_{1}} \times \cdots \times a_{l(\lambda)} \Delta_{\lambda_{l(\lambda)}}$. Consequently, it has Ehrhart polynomial

$$
i\left(\mathcal{F}_{(\lambda, \mathbf{a})}^{\lim }, t\right)=\prod_{j \in[\ell(\lambda)]}\left(\begin{array}{c}
t a_{j}+\lambda_{j} \\
\lambda_{j}
\end{array}\right)
$$

and normalized volumed

$$
\operatorname{vol} \mathcal{F}_{(\lambda, \mathbf{a})}^{\lim }=|\lambda| ! \prod_{i \in[\ell(\lambda)]} \frac{a_{i}^{\lambda_{i}}}{\lambda_{i} !}
$$

Theorem 16. Let $\lambda=\left(\lambda_{1}, \ldots, \lambda_{k}\right)$ be a partition, $n$ an integer such that $n-i \geqslant \lambda_{i}$ for all $i \in[\ell(\lambda)]$, and $\mathbf{a} \in \mathbb{Z}_{>0}^{n}$ a netflow vector. The face posets of the polytopes belonging to $\mathcal{F}_{(\lambda, \mathbf{a})}$ are isomorphic to the face poset of the Cartesian product of simplices $\Delta_{\lambda_{1}} \times \Delta_{\lambda_{2}} \times \cdots \times \Delta_{\lambda_{k}}$. In particular, the h-polynomial of the polytopes belonging to $\mathcal{F}_{(\lambda, \mathbf{a})}$ is

$$
\sum_{i=0}^{|\lambda|} h_{i} x^{i}=\prod_{i=1}^{k}\left[\lambda_{i}\right]_{x}=\prod_{i=1}^{k}\left(\sum_{j=0}^{\lambda_{i}-1} x^{j}\right)
$$


In particular, we see that Theorem 2 is a special case of Theorem 16 for $\mathcal{F}_{K_{n+1}}(\mathbf{1})$ which belongs to $\mathcal{F}_{((n-1, n-2, \ldots, 1), \mathbf{1})}$. Also notice the similar volumes for $\mathcal{F}_{K_{n+1}}(\mathbf{1})$ (Theorem 1) and $\mathcal{F}_{((n-1, n-2, \ldots, 1), \mathbf{1})}($ Theorem 8$)$; they are off by a factor of $\frac{2\left(\begin{array}{c}n \\ 2\end{array}\right)}{n !}$. We spell this curious fact out in the next corollary.

Corollary 3. $\operatorname{vol} \mathcal{F}_{K_{n+1}}(\mathbf{1})=\frac{2^{\left(\begin{array}{l}n \\ 2\end{array}\right)}}{n !} \operatorname{vol} \mathcal{F}_{((n-1, n-2, \ldots, 1), \mathbf{1})}^{\lim }$

The outline of this paper is as follows. In Section 2 we cover the necessary background and define the class $\mathcal{F}_{(\lambda, \mathbf{a})}$. In Section 3 we define the limiting polytope $\mathcal{F}_{(\lambda, \mathbf{a})}^{\lim }$ and prove Theorem 8. Section 4 is devoted to proving Theorem 16.

\section{Background and definitions}

\subsection{Flow polytopes and Kostant partition functions.}

The exposition of this section follows that of [4]; see [4] for more details.

Let $G$ be a (loopless) graph on the vertex set $[n+1]$ with $N$ edges. To each edge $(i, j), i<j$, of $G$, associate the positive type $A_{n}$ root $\mathrm{v}(i, j)=\mathbf{e}_{i}-\mathbf{e}_{j}$, where $\mathbf{e}_{i}$ is the $i$ th standard basis vector in $\mathbb{R}^{n+1}$. Let $S_{G}:=\left\{\left\{\mathbf{v}_{1}, \ldots, \mathbf{v}_{N}\right\}\right\}$ be the multiset of roots corresponding to the multiset of edges of $G$. Let $M_{G}$ be the $(n+1) \times N$ matrix whose columns are the vectors in $S_{G}$. Fix an integer vector $\mathbf{a}=\left(a_{1}, \ldots, a_{n+1}\right) \in \mathbb{Z}^{n+1}$ which we call the netflow and for which we require that $a_{n+1}=-\sum_{i=1}^{n} a_{i}$. An a-flow $\mathbf{f}_{G}$ on $G$ is a vector $\mathbf{f}_{G}=\left(b_{k}\right)_{k \in[N]}, b_{k} \in \mathbb{R}_{\geqslant 0}$ such that $M_{G} \mathbf{f}_{G}=\mathbf{a}$. That is, for all $1 \leqslant i \leqslant n+1$, we have

$$
\sum_{e=(g<i) \in E(G)} b(e)+a_{i}=\sum_{e=(i<j) \in E(G)} b(e)
$$

Define the flow polytope $\mathcal{F}_{G}(\mathbf{a})$ associated to a graph $G$ on the vertex set $[n+1]$ and the integer vector $\mathbf{a}=\left(a_{1}, \ldots, a_{n+1}\right)$ as the set of all a-flows $\mathbf{f}_{G}$ on $G$, i.e., $\mathcal{F}_{G}=\left\{\mathbf{f}_{G} \in \mathbb{R}_{\geqslant 0}^{N} \mid\right.$ $M_{G} \mathbf{f}_{G}=\mathbf{a}$ \}. The flow polytope $\mathcal{F}_{G}(\mathbf{a})$ then naturally lives in $\mathbb{R}^{N}$, where $N$ is the number of edges of $G$. Note that in order for $\mathcal{F}_{G}(\mathbf{a})$ to be nonempty, it must be that $\sum_{i=1}^{n+1} a_{i}=0$. For this reason, we also write $\mathcal{F}_{G}\left(a_{1}, \ldots, a_{n}\right):=\mathcal{F}_{G}\left(a_{1}, \ldots, a_{n},-\sum_{i=1}^{n} a_{i}\right)$. The vertices of the flow polytope $\mathcal{F}_{G}(\mathbf{a})$ are the a-flows whose supports are acyclic subgraphs of $G$ [3, Lemma 2.1].

Recall that the Kostant partition function $K_{G}$ evaluated at the vector $\mathbf{b} \in \mathbb{Z}^{n+1}$ is defined as

$$
K_{G}(\mathbf{b})=\#\left\{\left(c_{k}\right)_{k \in[N]} \mid \sum_{k \in[N]} c_{k} \mathbf{v}_{k}=\mathbf{b} \text { and } c_{k} \in \mathbb{Z}_{\geqslant 0}\right\},
$$

where $[N]=\{1,2, \ldots, N\}$.

The generating series of the Kostant partition function is

$$
\sum_{\mathbf{b} \in \mathbb{Z}^{n+1}} K_{G}(\mathbf{b}) \mathbf{x}^{\mathbf{b}}=\prod_{(i, j) \in E(G)}\left(1-x_{i} x_{j}^{-1}\right)^{-1}
$$


where $\mathbf{x}^{\mathbf{b}}=x_{1}^{b_{1}} x_{2}^{b_{2}} \cdots x_{n+1}^{b_{n+1}}$. In particular,

$$
K_{K_{n+1}}(\mathbf{b})=\left[\mathbf{x}^{\mathbf{b}}\right] \prod_{1 \leqslant i<j \leqslant n+1}\left(1-x_{i} x_{j}^{-1}\right)^{-1} .
$$

Assume that $\mathbf{a}=\left(a_{1}, a_{2}, \ldots, a_{n}\right)$ satisfies $a_{i} \geqslant 0$ for $i=1, \ldots, n$ and let

$\mathbf{a}^{\prime}=\left(a_{1}, a_{2}, \ldots, a_{n},-\sum_{i=1}^{n} a_{i}\right)$. The generalized Lidskii formulas of Baldoni and Vergne state that for a graph $G$ on the vertex set $[n+1]$ with $N$ edges we have

Theorem 4. [1, Theorem 38$]$

$$
\operatorname{vol} \mathcal{F}_{G}\left(\mathbf{a}^{\prime}\right)=\sum_{\mathbf{i}}\left(\begin{array}{c}
N-n \\
i_{1}, i_{2}, \ldots, i_{n}
\end{array}\right) a_{1}^{i_{1}} \cdots a_{n}^{i_{n}} \cdot K_{G^{\prime}}\left(i_{1}-t_{1}^{G}, i_{2}-t_{2}^{G}, \ldots, i_{n}-t_{n}^{G}\right),
$$

and

$$
K_{G}\left(\mathbf{a}^{\prime}\right)=\sum_{\mathbf{i}}\left(\begin{array}{c}
a_{1}+t_{1}^{G} \\
i_{1}
\end{array}\right)\left(\begin{array}{c}
a_{2}+t_{2}^{G} \\
i_{2}
\end{array}\right) \cdots\left(\begin{array}{c}
a_{n}+t_{n}^{G} \\
i_{n}
\end{array}\right) \cdot K_{G^{\prime}}\left(i_{1}-t_{1}^{G}, i_{2}-t_{2}^{G}, \ldots, i_{n}-t_{n}^{G}\right)
$$

where both sums are over weak compositions $\mathbf{i}=\left(i_{1}, i_{2}, \ldots, i_{n}\right)$ of $N-n$ with $n$ parts which we denote as $\mathbf{i}=N-n, \ell(\mathbf{i})=n$. The graph $G^{\prime}$ is the restriction of $G$ to the vertex set $[n]$. The notation $t_{i}^{G}, i \in[n]$, stands for the outdegree of vertex $i$ in $G$ minus 1 .

The notation vol stands for normalized volume. Recall that the Ehrhart polynomial $i(\mathcal{P}, t)$ of an integer polytope $\mathcal{P} \subset \mathbb{R}^{m}$ counts the number of integer points of dilations of the polytope, $i(\mathcal{P}, t):=\#\left(t \mathcal{P} \cap \mathbb{Z}^{m}\right)$. Its leading coefficient is the volume of the polytope. The normalized volume $\operatorname{vol}(P)$ of a $d$-dimensional polytope $\mathcal{P} \subset \mathbb{R}^{m}$ is the volume form which assigns a volume of one to the smallest $d$-dimensional integer simplex in the affine span of $\mathcal{P}$. In other words, the normalized volume of a $d$-dimensional polytope $\mathcal{P}$ is $d$ ! times its volume.

\subsection{The family $\mathcal{F}_{(\lambda, a)}$}

We start by defining a family of graphs associated to the partition $\lambda$. Given a partition $\lambda$, let $Y$ be the right-justified Young diagram corresponding to $\lambda$. Pick an integer $n$ such that $n-i \geqslant \lambda_{i}$ for all $i \in[\ell(\lambda)]$. We can place $Y$ inside the upper triangle (not including the diagonal) of an $n \times n$ matrix $M$, with the top and right edges of $Y$ flush with the top and right edges of $M$. Now, let $Y^{\prime}$ be the set of entries $(i, j)$ of $M$ that lie inside $Y$, and define $G(\lambda, n)$ to be the directed graph

$$
G(\lambda, n):=\left([n+1],\{(i, n+1): i \in[n]\} \cup Y^{\prime}\right) .
$$

Example 5. Construction of $G((2,1,1), 5))$. 

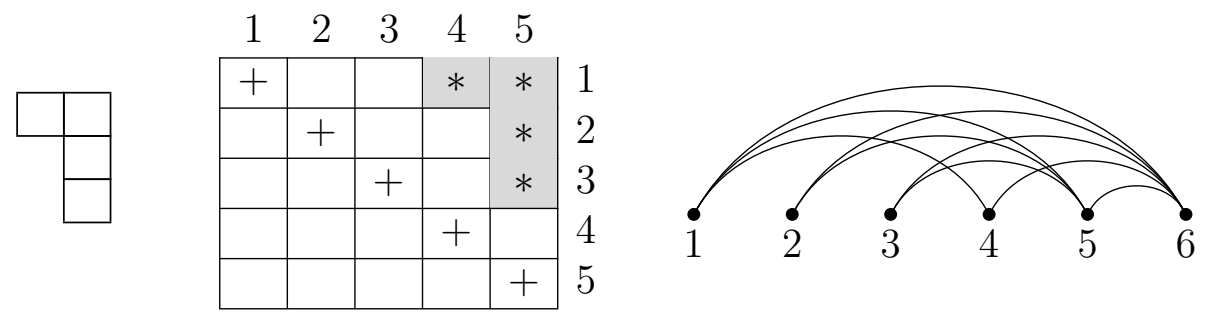

Figure 1: From left to right: the right-justified Young diagram of $\lambda=(2,1,1)$, the diagram in a $5 \times 5$ matrix, and the corresponding graph on six vertices.

For a fixed partition $\lambda$ and a fixed vector $\mathbf{a} \in \mathbb{Z}_{>0}^{m}$ with $m \geqslant \lambda_{1}+\ell(\lambda)$, define the family

$$
\mathcal{F}_{(\lambda, \mathbf{a})}:=\left\{\mathcal{F}_{G(\lambda, n)}(\mathbf{a}): \max \left(\lambda_{1}, \ell(\lambda)\right)<n \in \mathbb{Z}\right\} .
$$

Note that there is a small abuse of notation in the definition above: if $n \neq m$, then a will have too many or too few entries to serve as a netflow for many $G(\lambda, n)$. When $n \leqslant m$, then we can just use the first $n$ entries of a. For $n>m$, we show in Section 3 that the choice of additional entries is irrelevant: any element of $\mathbb{Z}_{>0}^{n}$ whose first $m$ entries match those of a will product essentially the same polytope. More precisely, we prove that all the above mentioned polytopes are integrally equivalent. Recall that integer polytopes $\mathcal{P} \subset \mathbb{R}^{m}$ and $\mathcal{Q} \subset \mathbb{R}^{k}$ are integrally equivalent if there is an affine transformation $f: \mathbb{R}^{m} \rightarrow \mathbb{R}^{k}$ such that $f$ maps $\mathcal{P}$ bijectively onto $\mathcal{Q}$ and $f$ maps $\mathbb{Z}^{m} \cap$ aff $(\mathcal{P})$ bijectively onto $\mathbb{Z}^{k} \cap$ aff $(\mathcal{Q})$, where aff denotes affine span. If two polytopes are integrally equivalent, then they have the same combinatorial type as well as the same volume and more generally the same Ehrhart polynomial.

Observe that for any $n \in \mathbb{Z}_{>0}$ and $\lambda=(n-1, n-2, \ldots, 1), G(\lambda, n)=K_{n+1}$. Setting $\mathbf{a}=\mathbf{1}$, it follows that the Tesler polytope $\mathcal{F}_{K_{n+1}}(\mathbf{1})$ belongs to $\mathcal{F}_{((n-1, n-2, \ldots, 1), \mathbf{1})}$.

\section{The limiting polytopes of $\mathcal{F}_{(\lambda, \mathrm{a})}$}

In this section we define the limiting polytope of the family $\mathcal{F}_{(\lambda, \mathbf{a})}$ for any partition $\lambda$ and netflow vector a. We then establish the combinatorial structure and the volume of these limiting polytopes.

One can easily see the need to define a limiting polytope of $\mathcal{F}_{(\lambda, \mathbf{a})}$ from the following data on the normalized volumes of the members of the family $\mathcal{F}_{((4,3,2,1), \mathbf{1})}$ :

$$
\begin{array}{r|lllllll}
n & 5 & 6 & 7 & 8 & 9 & 10 & 11 \\
\operatorname{vol} \mathcal{F}_{G((4,3,2,1), n)}(\mathbf{1}) & 107520 & 26580 & 15120 & 12600 & 12600 & 12600 & 12600
\end{array}
$$

One immediately notices that the volume of the polytopes in question appears to stabilize for large $n$. This is not a coincidence, and is in fact a general feature of polytopes in $\mathcal{F}_{(\lambda, \mathbf{a})}$, as we show in this section.

For a partition $\lambda$ and $\mathbf{a} \in \mathbb{Z}_{>0}^{n}$, define the limiting polytope of the family $\mathcal{F}_{(\lambda, \mathbf{a})}$, denoted $\mathcal{F}_{(\lambda, \mathbf{a})}^{\lim }$, to be the polytope $\mathcal{F}_{G\left(\lambda, \ell(\lambda)+\lambda_{1}\right)}(\mathbf{a})$. We prove in Lemma 6 that for all 
$n \geqslant \ell(\lambda)+\lambda_{1}$ we have that $\mathcal{F}_{G(\lambda, n)}(\mathbf{a})$ and $\mathcal{F}_{G\left(\lambda, \ell(\lambda)+\lambda_{1}\right)}(\mathbf{a})$ are integrally equivalent; thus any one of $\mathcal{F}_{G(\lambda, n)}(\mathbf{a})$ with $n \geqslant \ell(\lambda)+\lambda_{1}$ can be thought of as $\mathcal{F}_{(\lambda, \mathbf{a})}^{\lim }$.

\subsection{Structure and Volume of the Limiting Polytope}

Given a graph $G=G(\lambda, n)$, for each vertex $i \in[n]$, let

$$
G_{i}=([n+1],\{(i, j) \in E(G): i<j\} \cup\{(j, n+1): j \in[n]\})
$$

be the subgraph of $G$ graph obtained by restricting $E(G)$ to those edges that come out of vertex $i$ or go to the sink.

Lemma 6. Let $\lambda$ be a partition, let $n \geqslant \ell(\lambda)+\lambda_{1}$, let $G$ and $G_{i}$ be as above for $i \in[n]$, and let $\mathbf{a} \in \mathbb{Z}_{>0}^{n}$. Then $\mathcal{F}_{G(\lambda, n)}(\mathbf{a})$ is integrally equivalent to $\prod_{i=1}^{n} \mathcal{F}_{G_{i}}(\mathbf{a})$ :

$$
\mathcal{F}_{G(\lambda, n)}(\mathbf{a}) \equiv \prod_{i=1}^{n} \mathcal{F}_{G_{i}}(\mathbf{a})
$$

Proof. Define the map $\varphi: \mathcal{F}_{G}(\mathbf{a}) \rightarrow \prod_{i=1}^{n} \mathcal{F}_{G_{i}}(\mathbf{a})$ by

$$
\varphi(f)=\left(f_{1}, \ldots, f_{n}\right)
$$

where $f_{i}: E\left(G_{i}\right) \rightarrow \mathbb{R}$ is defined by

$$
f_{i}(p, q)= \begin{cases}f(i, j), & (p, q)=(i, j) \\ a_{p}+f(i, p), & q=n+1 \text { and }(i, p) \in E\left(G_{i}\right) \\ a_{p}, & q=n+1 \text { and }(i, p) \notin E\left(G_{i}\right)\end{cases}
$$

The inverse of map $\varphi$ is $\varphi^{-1}: \prod_{i=1}^{n} \mathcal{F}_{G_{i}} \rightarrow \mathcal{F}_{G}$ defined by $\varphi^{-1}\left(f_{1}, \ldots, f_{n}\right)=f$ where $f(p, q)=f_{p}(p, q)$, thus $\varphi$ is a bijection between $\mathcal{F}_{G(\lambda, n)}(\mathbf{a})$ and $\prod_{i=1}^{n} \mathcal{F}_{G_{i}}(\mathbf{a})$. Moreover, $\varphi$ can be extended to an affine map mapping the integer points of the affine span of $\mathcal{F}_{G(\lambda, n)}(\mathbf{a})$ bijectively to the integer points of the affine span of $\prod_{i=1}^{n} \mathcal{F}_{G_{i}}(\mathbf{a})$, concluding the proof.

We now show that the polytopes $\mathcal{F}_{G_{i}}(\mathbf{a})$ appearing in Lemma 6 are very special. Denote by $\Delta_{m}$ the standard simplex of dimension $m$, the convex hull of the standard basis vectors of $\mathbb{R}^{m+1}$.

Lemma 7. For $i \in[\ell(\lambda)], \mathbf{a} \in \mathbb{Z}_{>0}^{n}, \mathcal{F}_{G_{i}}(\mathbf{a})$ is integrally equivalent to $a_{i} \Delta_{\lambda_{i}}$, a scaled simplex of dimension $\lambda_{i}$. For $\ell(\lambda)<i<n+1, \mathcal{F}_{G_{i}}(\mathbf{a})$ is a point.

Proof. Let $i \in[\ell(\lambda)]$. Define $\varphi_{i}: \mathcal{F}_{G_{i}}(\mathbf{a}) \rightarrow a_{i} \Delta_{\lambda_{i}}$ by $\varphi_{i}\left(f_{i}\right)=\mathbf{v} \in \mathbb{R}^{\lambda_{i}+1}$, where $v_{j}=$ $f_{i}(i, n+2-j)$. To see that this function is well-defined, note that $i \in V\left(G_{i}\right)$ has no incoming edges (see Figure 2), so $\sum_{(i, j) \in E\left(G_{i}\right)} f(i, j)=\sum_{j \in\left[\lambda_{i}+1\right]} v_{j}=a_{i}$. This map is a projection; it is affine and preserves integer points. It is not hard to see that $\varphi_{i}$ is a bijection between $\mathcal{F}_{G_{i}}(\mathbf{a})$ and $a_{i} \Delta_{\lambda_{i}}$. Furthermore, the second claim of Lemma 7 is immediate. 
Lemmas 6 and 7 imply that we can consider any polytope in $\mathcal{F}_{G(\lambda, n)}(\mathbf{a})$ with $n \geqslant$ $\ell(\lambda)+\lambda_{1}$ as the limiting polytope $\mathcal{F}_{(\lambda, \mathbf{a})}^{\lim }$. Indeed, when $n \geqslant \lambda_{1}+\ell(\lambda)$, it is guaranteed that the Young diagram of $\lambda$ will fit in the top right quadrant of an $n \times n$ matrix. Figure 2 illustrates the effects of this.
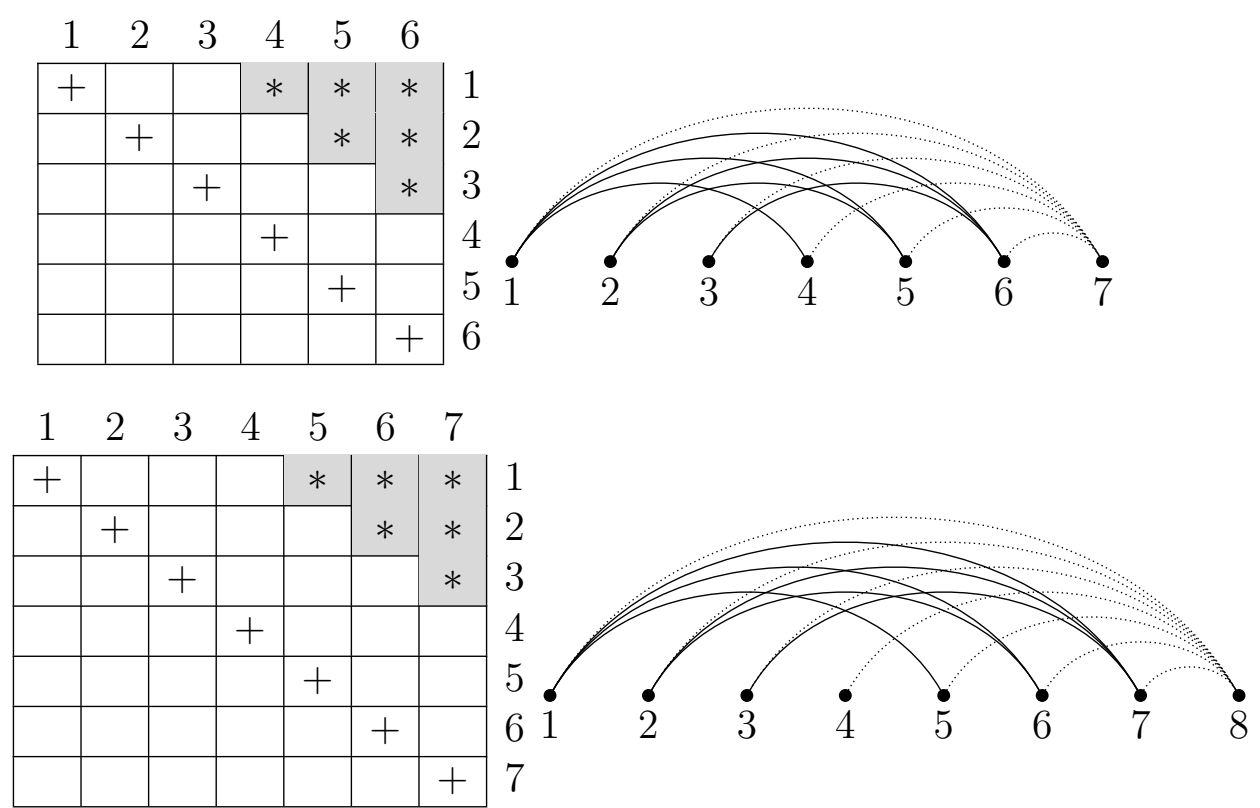

Figure 2: The Young diagram of $\lambda=(3,2,1)$ in both $6 \times 6$ and $7 \times 7$ matrices, and the corresponding graphs $G(\lambda, 6)$ and $G(\lambda, 7)$, with edges to the sink dotted. Observe that increasing $n$ by 1 adds a single new vertex with a single outgoing edge to the sink. This underlies the fact that $\mathcal{F}_{G(\lambda, 6)}(\mathbf{a})$ and $\mathcal{F}_{G(\lambda, 7)}(\mathbf{a})$ are integrally equivalent and have the same volume. It also justifies our use of a as the netflow vector for both $\mathcal{F}_{G(\lambda, 6)}$ and $\mathcal{F}_{G(\lambda, 7)}$ : only the first $\ell(\lambda)$ entries of the netflow vector matter.

The decomposition of the limiting polytope into simplices also gives us a neat formula for its volume.

Theorem 8. Let $\lambda$ be a partition, $n \geqslant \lambda_{1}+\ell(\lambda)$, and $\mathbf{a} \in\left(\mathbb{Z}_{>0}\right)^{n}$ a vector of positive integers. The limiting polytope of $\mathcal{F}_{(\lambda, \mathbf{a})}$ is integrally equivalent to a product of scaled simplices $a_{1} \Delta_{\lambda_{1}} \times \cdots \times a_{l(\lambda)} \Delta_{\lambda_{l(\lambda)}}$. Consequently, it has Ehrhart polynomial

$$
i\left(\mathcal{F}_{(\lambda, \mathbf{a})}^{\lim }, t\right)=\prod_{j \in[\ell(\lambda)]}\left(\begin{array}{c}
t a_{j}+\lambda_{j} \\
\lambda_{j}
\end{array}\right)
$$

and normalized volumed

$$
\operatorname{vol} \mathcal{F}_{(\lambda, \mathbf{a})}^{\lim }=|\lambda| ! \prod_{i \in[\ell(\lambda)]} \frac{a_{i}^{\lambda_{i}}}{\lambda_{i} !}
$$

Proof. It is immediate from Lemmas 6 and 7 that $\mathcal{F}_{(\lambda, \mathbf{a})}^{\lim }$ is integrally equivalent to 
$\prod_{i \in[\ell(\lambda)]} a_{i} \Delta_{\lambda_{i}}$. From integral equivalence, it follows that

$$
i\left(\mathcal{F}_{(\lambda, \mathbf{a})}^{\lim }, t\right)=\prod_{i \in[\ell(\lambda)]} i\left(a_{i} \Delta_{\lambda_{i}}, t\right)
$$

It is well-known that $i\left(a_{i} \Delta_{\lambda_{i}}, t\right)=\left(\begin{array}{c}t a_{i}+\lambda_{i} \\ \lambda_{i}\end{array}\right)$, so the Ehrhart polynomial of $\mathcal{F}_{(\lambda, \mathbf{a})}^{\lim }$ is as claimed.

To prove the stated formula for volume, recall that the unnormalized relative volume of a polytope is given by the leading coefficient of its Ehrhart polynomial. Computing the leading coefficient of $i\left(\mathcal{F}_{(\lambda, \mathbf{a})}^{\lim }, t\right)$ using the formula given above and multiplying it by $\left(\operatorname{dim} \mathcal{F}_{(\lambda, \mathbf{a})}^{\lim }\right) !=|\lambda|$ ! to normalize it yields a (normalized) volume of

$$
\operatorname{vol} \mathcal{F}_{(\lambda, \mathbf{a})}^{\lim }=|\lambda| ! \prod_{i \in \ell(\lambda)} \frac{a_{i}^{\lambda_{i}}}{\lambda_{i} !}
$$

We note that we can relax the requirement $\mathbf{a} \in\left(\mathbb{Z}_{>0}\right)^{n}$ to $\mathbf{a} \in\left(\mathbb{Z}_{\geqslant 0}\right)^{n}$ and obtain similar results. Indeed, both Lemmas 6 and 7 and their proofs hold verbatim (if $a_{i}=0$ then $\mathcal{F}_{G_{i}}(\mathbf{a})$ is a point). Thus, the analogues of Lemmas 6 and 7 yield a volume formula for any $\mathcal{F}_{(\lambda, \mathbf{a})}^{\lim }, \mathbf{a} \in\left(\mathbb{Z}_{\geqslant 0}\right)^{n}$. For simplicity, we will work with $\mathbf{a} \in\left(\mathbb{Z}_{>0}\right)^{n}$ throughout the paper.

\subsection{Constant Term Identities}

Using the volume formula given in Theorem 8, we can derive a constant term identity. Let $\lambda$ be a partition, $n$ an integer such that $n-i \geqslant \lambda_{i}$ for all $i \in[\ell(\lambda)]$, and $\mathbf{a} \in \mathbb{Z}_{>0}^{n}$. For convenience, let $L=|\lambda|$ and let $G$ be the restriction of $G(\lambda, n)$ to the vertex set $[n]$. Further, let $\bar{\lambda}=\left(\lambda_{1}, \ldots, \lambda_{\ell(\lambda)}, 0, \ldots, 0,0\right) \in \mathbb{Z}^{n}$.

\section{Theorem 9.}

$$
C T_{x_{n}} \ldots \mathrm{CT}_{x_{1}}\left(a_{1} x_{1}+\cdots+a_{n} x_{n}\right)^{L} \prod_{i \in[\ell(\lambda)]} \prod_{n+1-\lambda_{i} \leqslant j \leqslant n}\left(x_{i}-x_{j}\right)^{-1}=L ! \prod_{i \in[\ell(\lambda)]} \frac{a_{i}^{\lambda_{i}}}{\lambda_{i} !}
$$

Proof. By Equation (2.5), the volume of $\mathcal{F}_{(\lambda, \mathbf{a})}^{\lim }$ is equal to

$$
\operatorname{vol} \mathcal{F}_{(\lambda, \mathbf{a})}^{\lim }=\sum_{\substack{\mathbf{i} \in L \\
\ell(\mathbf{i})=n}}\left(\begin{array}{c}
L \\
i_{1}, \ldots, i_{n}
\end{array}\right)\left(\prod_{j \in[n]} a_{j}^{i_{j}}\right) K_{G}(\mathbf{i}-\bar{\lambda})
$$

Now, let $G^{\prime}$ be $G$ with all its edges reversed and observe that $K_{G}(\mathbf{i}-\bar{\lambda})=K_{G^{\prime}}(\bar{\lambda}-\mathbf{i})$. 
Thus, the above is equal to

$$
\begin{aligned}
& =\sum_{\substack{\mathbf{i} \in L \\
\ell(\mathbf{i})=n}}\left(\begin{array}{c}
L \\
i_{1}, \ldots, i_{n}
\end{array}\right)\left(\prod_{j \in[n]} a_{j}^{i_{j}}\right) K_{G^{\prime}}(\bar{\lambda}-\mathbf{i}) \\
& =\sum_{\substack{\mathbf{i} \in L \\
\ell(\mathbf{i})=n}}\left(\begin{array}{c}
L \\
i_{1}, \ldots, i_{n}
\end{array}\right)\left(\prod_{j \in[n]} a_{j}^{i_{j}}\right)\left[\mathbf{x}^{\bar{\lambda}-\mathbf{i}}\right] \prod_{(i, j) \in E(G)}\left(1-x_{j} x_{i}^{-1}\right)^{-1} \\
& =\mathrm{CT}_{x_{n}} \ldots \mathrm{CT}_{x_{1}} \sum_{\substack{\mathbf{i} \in L \\
\ell(\mathbf{i})=n}}\left(\begin{array}{c}
L \\
i_{1}, \ldots, i_{n}
\end{array}\right)\left(\prod_{j \in[n]} a_{j}^{i_{j}}\right) \mathbf{x}^{\mathbf{i}-\bar{\lambda}} \prod_{(i, j) \in E(G)}\left(1-x_{j} x_{i}^{-1}\right)^{-1} .
\end{aligned}
$$

Since the $i$ th vertex of $G$ has $\bar{\lambda}_{i}$ edges out of it,

$$
\prod_{(i, j) \in E(G)}\left(1-x_{j} x_{i}^{-1}\right)^{-1}=\mathbf{x}^{\bar{\lambda}} \prod_{(i, j) \in E(G)}\left(x_{i}-x_{j}\right)^{-1} .
$$

It follows that the above is equal to

$$
\begin{aligned}
& =\mathrm{CT}_{x_{n}} \ldots \mathrm{CT}_{x_{1}} \sum_{\substack{\mathbf{i} \in L \\
\ell(\mathbf{i})=n}}\left(\begin{array}{c}
L \\
i_{1}, \ldots, i_{n}
\end{array}\right)\left(\prod_{j \in[n]} a_{j}^{i_{j}}\right) \mathrm{x}^{\mathbf{i}} \prod_{(i, j) \in E(G)}\left(x_{i}-x_{j}\right)^{-1} \\
& =\mathrm{CT}_{x_{n}} \ldots \mathrm{CT}_{x_{1}}\left(a_{1} x_{1}+\cdots+a_{n} x_{n}\right)^{L} \prod_{(i, j) \in E(G)}\left(x_{i}-x_{j}\right)^{-1}
\end{aligned}
$$

where the latter equality follows by the multinomial theorem. The product in the last expression can be rewritten as $\prod_{i \in[\ell(\lambda)]} \prod_{n+1-\lambda_{i} \leqslant j \leqslant n}\left(x_{i}-x_{j}\right)^{-1}$. Finally, substituting in the formula for $\operatorname{vol} \mathcal{F}_{(\lambda, \mathbf{a})}^{\lim }$ given in Theorem 8 yields the result.

\section{The face structure of polytopes in $\mathcal{F}_{(\lambda, \mathrm{a})}$}

In Theorem 8, we showed that for all $\lambda$ and $\mathbf{a} \in \mathbb{Z}_{>0}^{n}, \mathcal{F}_{(\lambda, \mathbf{a})}^{\lim }$ is integrally equivalent to a product of simplices, implying that its combinatorial type is that of a product of simplices. In this section, we show that each element of the family $\mathcal{F}_{(\lambda, \mathbf{a})}^{\lim }$ has the same combinatorial type as $\mathcal{F}_{(\lambda, \mathbf{a})}^{\lim }$.

\subsection{A quick review of results relating subgraphs and the face lattice}

Before proceeding, we will review some facts relating the face lattice of a flow polytope to subgraphs of the graph from which it arises.

Let $G$ be a graph and a netflow vector. We call a subgraph $H$ of $G$ a-regular (or just regular when the netflow in question is clear) if there is an a-flow $f$ on $G$ such that 
$f$ is zero on all edges of $G$ that are not in $H$. We say that a is in generic position with respect to $G$ if there is no a-flow $f$ such that $f$ is the unique flow on two distinct subtrees of $G$. The following two results are implied by [3, Lemma $2.1 \&$ Theorem 2.2] for the faces of $\mathcal{F}_{G_{(\lambda, n)}}(\mathbf{a})$.

Lemma 10. The vertices of $\mathcal{F}_{G_{(\lambda, n)}}(\mathbf{a})$ are the flows on the regular subtrees of $G_{(\lambda, n)}$.

Theorem 11. If $\mathbf{a}$ is in generic position, then the regular subtrees of $G_{(\lambda, n)}$ are in bijection with the vertices of $\mathcal{F}_{G_{(\lambda, n)}}(\mathbf{a})$ and the faces of $\mathcal{F}_{G_{(\lambda, n)}}(\mathbf{a})$ are in bijection with the regular subgraphs of $G_{(\lambda, n)}$.

\subsection{Characterization of regular subtrees}

Let $\lambda=\left(\lambda_{1}, \ldots, \lambda_{\ell}\right)$ be a partition, $n$ an integer such that $n-i \geqslant \lambda_{i}$ for all $i \in[\ell(\lambda)]$, $G=G(\lambda, n)$, and let $\mathbf{a} \in \mathbb{Z}_{>0}^{n}$. In this section, we characterize which subtrees of $G$ are a-regular for $\mathbf{a} \in \mathbb{Z}_{>0}^{n}$.

Lemma 12. Let $H$ be a subgraph of $G$ built by picking one outgoing edge from each vertex $i<n+1$. Then $H$ is an a-regular spanning tree of $G$ for $\mathbf{a} \in \mathbb{Z}_{>0}^{n}$.

Proof. First we show that $H$ is acyclic and connected. If there were a cycle $C \subset H$, then there would have to be two outgoing edges from its minimal vertex. Thus, $H$ is acyclic. To see that $H$ is connected, we note that our graph has $n$ edges, $n+1$ vertices and it has no cycles.

To see that $H$ is regular, construct an a-flow on it as follows. Let $e_{v} \in E(H)$ be the unique edge out of $v$ in $H$. Let $f\left(e_{1}\right)=a_{1}$. Assume $f\left(e_{1}\right), \ldots, f\left(e_{i}\right)$ have been assigned for some $i \geqslant 1$. Then we let $f\left(e_{i+1}\right)=a_{i+1}$ if $i+1$ has no incoming edges in $H$, and $f\left(e_{i+1}\right)=a_{i+1}+\sum_{(v, i+1) \in E(H)} f\left(e_{v}\right)$ for vertices with incoming edges.

Lemma 13. Every spanning subtree $T$ of $G$ that admits an $\mathbf{a}$-flow for $\mathbf{a} \in \mathbb{Z}_{>0}^{n}$ has a single edge out of each of its vertices $v<n+1$.

Proof. Suppose that a spanning subtree $T$ of $G$ has at least two outgoing edges from a vertex $v$. Since $T$ has $n$ edges and $n+1$ vertices, it follows then that $T$ has two vertices with no outgoing edges. In particular, there is a $v<n+1$ with only incoming edges. Since $a_{v}>0$, such a tree cannot admit an a-flow. Thus each spanning subtree $T$ of $G$ that admits an a-flow has at most one edge out of each of its vertices $v<n+1$. Since we need $n$ edges, it has exactly one edge out of each of its vertices $v<n+1$.

Theorem 14. The a-regular subtrees for $\mathbf{a} \in \mathbb{Z}_{>0}^{n}$ of $G(\lambda, n)$ are precisely those that have exactly one edge out of every vertex. The polytope $\mathcal{F}_{G(\lambda, n)}(\mathbf{a})$ has $\prod_{i=1}^{\ell(\lambda)}\left(\lambda_{i}+1\right)$ vertices, independent of $n$, corresponding to the a-flows on the aforementioned subtrees.

Proof. The first statement follows immediately from Lemmas 12 and 13 . We can count such trees by noting that each vertex $i$ has $1+\lambda_{i}$ edges out of it (and 1 edge if $i>\ell$ ), so there are $\prod_{i=1}^{\ell}\left(\lambda_{i}+1\right)$ ways to choose such a tree. 
Now, note that if $T$ is a spanning tree of $G$ that admits a flow $f_{T}$, then $f_{T}$ must be nonzero on every edge of $T$ because a's non-sink entries are all positive. Thus, a is in generic position and by Lemma 11 there is a bijection between subtrees of $G$ that admit regular flows and vertices of $\mathcal{F}_{G}(\mathbf{a})$.

\subsection{The face lattice}

We are ready to show that every polytope in $\mathcal{F}_{(\lambda, \mathbf{a})}$ has a face lattice isomorphic to that of $\mathcal{F}_{(\lambda, \mathbf{a})}^{\lim }$.

Lemma 15. Let $\lambda$ be a partition, $n$ an integer such that $n-i \geqslant \lambda_{i}$ for all $i \in[\ell(\lambda)]$, and $\mathbf{a} \in \mathbb{Z}_{>0}^{n}$. The regular subgraphs of $G(\lambda, n)$ are precisely those that have at least one edge out of every non-sink vertex. Furthermore, for $H$ and $K$ regular subgraphs of $G(\lambda, n)$, $\mathcal{F}_{K}(\mathbf{a}) \subset \mathcal{F}_{H}(\mathbf{a})$ if and only if $K$ is a subgraph of $H$. Thus, the face lattice of $\mathcal{F}_{G(\lambda, n)}(\mathbf{a})$ is isomorphic to the poset of regular subgraphs of $G(\lambda, n)$.

Proof. The entries of a are all positive, so every vertex of a regular subgraph must have at least one outgoing edge. Conversely, any subgraph that has at least one edge out of every non-sink vertex contains a regular subtree by Theorem 14 and is therefore regular.

For the second statement, the "if" implication is clear. For the "only if", observe that if $e$ is an edge in $K$ that is not in $H$, then there is a regular subtree $T$ contained in $K$ such that $e \in E(T)$. Since $\mathbf{a}$ is in generic position, the unique flow $f$ on $T$ is nonzero on $e$ and is therefore not in $\mathcal{F}_{H}(\mathbf{a})$, so $\mathcal{F}_{K}(\mathbf{a}) \not \subset \mathcal{F}_{H}(\mathbf{a})$. The last statement then follows by Lemma 11 since $\mathbf{a}$ is in generic position.

Theorem 16. Let $\lambda=\left(\lambda_{1}, \ldots, \lambda_{k}\right)$ be a partition, $n$ an integer such that $n-i \geqslant \lambda_{i}$ for all $i \in[\ell(\lambda)]$, and $\mathbf{a} \in \mathbb{Z}_{>0}^{n}$ a netflow vector. The face posets of the polytopes belonging to $\mathcal{F}_{(\lambda, \mathbf{a})}$ are isomorphic to the face poset of the Cartesian product of simplices $\Delta_{\lambda_{1}} \times \Delta_{\lambda_{2}} \times \cdots \times \Delta_{\lambda_{k}}$. In particular, the h-polynomial of the polytopes belonging to $\mathcal{F}_{(\lambda, \mathbf{a})}$ is

$$
\sum_{i=0}^{|\lambda|} h_{i} x^{i}=\prod_{i=1}^{k}\left[\lambda_{i}\right]_{x}=\prod_{i=1}^{k}\left(\sum_{j=0}^{\lambda_{i}-1} x^{j}\right) .
$$

Proof. Let $\bar{\lambda}=\left(\lambda_{1}+1, \lambda_{2}+1, \ldots, \lambda_{k}+1\right)$ and let $Y$ be the Young diagram of $\bar{\lambda}$. Let $\mathcal{C}$ be the poset of subsets $C$ of the boxes of $Y$ such that $C$ contains at least one box from every row of $Y$, ordered by inclusion.

For any integer $n$ such that $n-i \geqslant \lambda_{i}$ for all $i \in[\ell(\lambda)]$, define the bijection $\varphi_{n}$ : $Y \rightarrow E(G(\lambda, n))$ by $\varphi_{n}(i, j)=(i, n+2-j)$. Now, let $\mathcal{R}(G(\lambda, n))$ be the set of regular subgraphs of $G$ and define $\phi_{n}: \mathcal{C} \rightarrow \mathcal{R}(G(\lambda, n))$ by

$$
C \mapsto\left([n+1], \varphi_{n}(C) \cup\{(i, n+1): n+1>i>\ell(\lambda)\}\right) .
$$

Every subgraph in the image of $\phi_{n}$ has an edge out of every vertex besides $n+1$, so by Lemma 15, the image of $\phi_{n}$ lies in $\mathcal{R}(G(\lambda, n))$ as claimed. In fact, using the surjectivity 
of $\varphi_{n}$, the image of $\phi_{n}$ is all of $\mathcal{R}(G(\lambda, n))$. Finally, injectivity of $\phi_{n}$ follows from the fact that $\varphi_{n}$ is injective, and it is clear that it preserves inclusion. Therefore, $\phi_{n}$ is an order preserving bijection between $\mathcal{C}$ and $\mathcal{R}(G(\lambda, n))$. Applying the second statement of Lemma 15 , we have that for any integer $n$ such that $n-i \geqslant \lambda_{i}$ for all $i \in[\ell(\lambda)]$, the face lattice of $\mathcal{F}_{G(\lambda, n)}(\mathbf{a})$ is isomorphic to $\mathcal{C}$. In particular, the face lattice of $\mathcal{F}_{(\lambda, \mathbf{a})}^{\lim }=\mathcal{F}_{G\left(\lambda, \lambda_{1}+\ell(\lambda)\right)}(\mathbf{a})$ is isomorphic to $\mathcal{C}$. The former is isomorphic to the face lattice of $\Delta_{\lambda_{1}} \times \Delta_{\lambda_{2}} \times \cdots \times \Delta_{\lambda_{k}}$ by Theorem 8. It follows that every element of $\mathcal{F}_{(\lambda, \mathbf{a})}$ has a face lattice isomorphic to this one. Furthermore, $\mathcal{F}_{(\lambda, \mathbf{a})}$ is of dimension $|\lambda|$ and its $h$-polynomial is given by the products of the $h$-polynomials of the simplices: $h\left(\mathcal{F}_{(\lambda, \mathbf{a})}, x\right)=\prod_{i=1}^{k} h\left(\Delta_{\lambda_{i}}, x\right)=\prod_{i=1}^{k}\left[\lambda_{i}\right]_{x}$, as desired.

Remark 17. Since $\mathcal{F}_{K_{n+1}}(\mathbf{1})$ is an element of $\mathcal{F}_{((n-1, n-2, \ldots, 1), \mathbf{1})}$, Theorem 2 is a special case of Theorem 16.

\section{References}

[1] W. Baldoni and M. Vergne. Kostant partitions functions and flow polytopes. Transform. Groups, 13(3-4):447-469, 2008.

[2] C.S. Chan, D.P. Robbins, and D.S. Yuen. On the volume of a certain polytope. Experiment. Math., 9(1):91-99, 2000.

[3] L. Hille. Quivers, cones and polytopes. Linear Algebra Appl., (365):215-237.

[4] K. Mészáros and A. H. Morales. Flow polytopes of signed graphs and the Kostant partition function. Int. Math. Res. Notices, (3):830-871, 2015.

[5] K. Mészáros, A.H. Morales, and B. Rhoades. The polytope of Tesler matrices. Selecta Mathematica, 23 (2017), no. 1, 425-454. arXiv:1409.8566v2.

[6] R. P. Stanley. Enumerative combinatorics. Vol. 2, volume 62 of Cambridge Studies in Advanced Mathematics. Cambridge University Press, Cambridge, 1999. With a foreword by Gian-Carlo Rota and appendix 1 by Sergey Fomin. 